\title{
Microbiome composition indicate dysbiosis and lower richness in tumor breast tissues compared to healthy adjacent paired tissue, within the same women
}

\author{
Maria Valeria Esposito ${ }^{1,2 \dagger}$, Bruno Fosso ${ }^{3 \dagger}$, Marcella Nunziato ${ }^{1,2 \dagger}$, Giorgio Casaburi ${ }^{4}$, Valeria D'Argenio ${ }^{1,2,5}$, \\ Alessandra Calabrese ${ }^{6}$, Massimiliano D'Aiuto ${ }^{6,7}$, Gerardo Botti $^{8}$, Graziano Pesole $^{3,9^{*}+}$ and \\ Francesco Salvatore ${ }^{1,2^{*}+}$ (B)
}

\begin{abstract}
Background: Breast cancer (BC) is the most common malignancy in women, in whom it reaches $20 \%$ of the total neoplasia incidence. Most BCs are considered sporadic and a number of factors, including familiarity, age, hormonal cycles and diet, have been reported to be BC risk factors. Also the gut microbiota plays a role in breast cancer development. In fact, its imbalance has been associated to various human diseases including cancer although a consequential cause-effect phenomenon has never been proven.

Methods: The aim of this work was to characterize the breast tissue microbiome in 34 women affected by BC using an NGS-based method, and analyzing the tumoral and the adjacent non-tumoral tissue of each patient.

Results: The healthy and tumor tissues differed in bacterial composition and richness: the number of Amplicon Sequence Variants (ASVs) was higher in healthy tissues than in tumor tissues $(p=0.001)$. Moreover, our analyses, able to investigate from phylum down to species taxa for each sample, revealed major differences in the two richest phyla, namely, Proteobacteria and Actinobacteria. Notably, the levels of Actinobacteria and Proteobacteria were, respectively, higher and lower in healthy with respect to tumor tissues.

Conclusions: Our study provides information about the breast tissue microbial composition, as compared with very closely adjacent healthy tissue (paired samples within the same woman); the differences found are such to have possible diagnostic and therapeutic implications; further studies are necessary to clarify if the differences found in the breast tissue microbiome are simply an association or a concausative pathogenetic effect in BC. A comparison of different results on similar studies seems not to assess a universal microbiome signature, but single ones depending on the environmental cohorts'locations.
\end{abstract}

\footnotetext{
*Correspondence: g.pesole@ibiom.cnr.it; salvator@unina.it

${ }^{\dagger}$ Maria Valeria Esposito, Bruno Fosso and Marcella Nunziato are Co-first

authors.

${ }^{\dagger}$ Graziano Pesole and Francesco Salvatore are Co-last authors.

${ }^{2}$ CEINGE - Biotecnologie Avanzate, Via Gaetano Salvatore, 486

80145 Napoli, Italy

9 Department of Biosciences, Biotechnology and Biopharmaceutics,

University of Bari "A. Moro", Piazza Umberto I, 1, BA 70121 Bari, Italy

Full list of author information is available at the end of the article
}

(C) The Author(s) 2021. Open Access This article is licensed under a Creative Commons Attribution 4.0 International License, which permits use, sharing, adaptation, distribution and reproduction in any medium or format, as long as you give appropriate credit to the original author(s) and the source, provide a link to the Creative Commons licence, and indicate if changes were made. The images or other third party material in this article are included in the article's Creative Commons licence, unless indicated otherwise in a credit line to the material. If material is not included in the article's Creative Commons licence and your intended use is not permitted by statutory regulation or exceeds the permitted use, you will need to obtain permission directly from the copyright holder. To view a copy of this licence, visit http://creativecommons.org/licenses/by/4.0/. The Creative Commons Public Domain Dedication waiver (http://creativeco mmons.org/publicdomain/zero/1.0/) applies to the data made available in this article, unless otherwise stated in a credit line to the data. 
Keywords: Breast cancer microbiome, Microbial dysbiosis, Breast cancer tissues, Next generation sequencing, Breast healthy tissues, Microbiome composition, cancer/healthy paired samples, 16S rRNA

\section{Background}

Breast cancer $(\mathrm{BC})$ is the most common form of cancer among women and, after ovarian cancer, is the second cause of death due to a neoplastic disease worldwide [1, 2]. Familial forms of BC represent up to $20 \%$ of all BCs: among these more than $25 \%$ are due to predisposing mutations in the BRCA1/2 genes [3-9] while another percentage concerns mutations in high, moderate and low susceptibility genes [10]. Despite this genetic component, the etiology of up to $80-85 \%$ of tumors remains unknown and thus they are considered sporadic. In this context, environmental and lifestyle factors might also modify cancer risk in both familial and sporadic BCs. Nevertheless, most of the factors contributing to $B C$ are still not completely understood thereby limiting $B C$ prevention and treatment measures $[11,12]$.

The human microbiome plays an important role in promoting health and preventing disease, which suggests that microbial dysbiosis could contribute to increasing the risk of cancer [13-19]. In this regard, in recent years attention has focused on the relationship between the human microbiome and carcinogenesis to assess its role in BC onset and/or development [20-24]. Therefore, in this scenario, we analyzed (in paired samples from the same subject) the microbiome of tumor breast tissue and the adjacent normal one of women affected by $\mathrm{BC}$ in the attempt to get a closer view which may shed light on the potential involvement of microbial dysbiosis in breast cancer. To this aim, we used next-generation-sequencing (NGS)-based methodology to analyze the $16 \mathrm{~s}$ ribosomal RNA of the microbiome tissue populations.

\section{Methods}

\section{Patients' samples and ethics}

Biological samples and clinical data were obtained from a total of 34 women attending the Breast Unit of the "Istituto Nazionale dei Tumori - Fondazione G. Pascale" of Naples starting in 2014 lasting 5 years (Table 1). All patients gave their written informed consent to the study that was carried out according to the tenets of the Helsinki Declaration and approved by the Istituto Nazionale Tumori - Fondazione G. Pascale Ethics Committee (protocol number 3 of 03/25/2009). All patients were previously screened for BRCA1/2 mutations using the protocol and the selection criteria reported by D'Argenio et al. 2015 [25].

Tumor tissues and healthy tissues, singly paired from the same woman, and surgically removed at the same time (within the same sequencing run, see below), were analyzed for a total of 68 samples, from which total DNA was isolated. Only fresh frozen tissues were used. The tissues were frozen immediately after removal directly in the surgery room to avoid environmental contamination.

To precisely ensure the histology of tissues, all were analyzed in the pathology laboratory (see Table 1).

\section{Genomic DNA extraction from breast tissue}

DNA was extracted from tissues using the QIAamp DNeasy Blood \& Tissue kits (Qiagen, Hilden, Germany), according to the manufacturer's instructions. DNA was quantified using the NanoDrop 2000c Spectrophotometer (Thermo Fisher Scientific, Waltham, MA, USA) and the Qubit dsDNA BR and HS assay kit (Life Technologies, CA, USA).

\section{Preparation of the $16 \mathrm{~S}$ Metagenomic Sequencing Library} Amplification of the V4-V6 regions of the $16 \mathrm{~S}$ rRNA bacterial genes was assessed in two PCR steps: a template of $5 \mathrm{ng} / \mu \mathrm{l}$ of DNA for each sample was used for the first PCR, which was performed using the V4-V6 region specific primers with overhang adapters attached. The primer sequences used in this study are listed in Table 2; the primers were designed and synthetized in our core facility.

Subsequently, $1 \mu \mathrm{l}$ of the PCR product was analyzed on a Bioanalyzer DNA 1000 chip (Agilent, Santa Clara, CA, USA) to verify its size ( $550 \mathrm{bp})$. Next, Agencourt AMPure XP beads (Beckman Coulter, Brea, CA, USA) were used to purify the $16 \mathrm{~S} \mathrm{V4-V6} \mathrm{amplicons} \mathrm{away} \mathrm{from}$ free primers and primer dimer species. Purification products underwent further quality and quantity controls by Bioanalyzer DNA 1000 analysis (Agilent, Santa Clara, CA, USA). The second PCR, performed as per the Nextera XT protocol (Illumina, San Diego, CA), allowed the addition of the Illumina sequencing adapters and the dual-index primers, which barcoded each sample. The V4-V6 amplified regions of each patient were purified through Agencourt AMPure XP Beads (Beckman Coulter, Brea, CA, USA), quantified using the Qubit HS assay kit (Life Technologies, CA, USA) and quality-assessed using a High Sensitivity Chip on the 2100 Bioanalyzer Instruments (Agilent, Santa Clara, CA, USA). However, up to 68 libraries were pooled together for sequencing. Therefore, $8 \mathrm{pM}$ of denatured libraries were combined to $25 \%$ of $8 \mathrm{pM}$ PhiX control and loaded into the MiSeq v3 reagent cartridge. Sequencing reactions were per-formed 
Table 1 Anamnestic and clinical features of patients selected for this study

\begin{tabular}{|c|c|c|c|c|c|c|c|c|}
\hline ID & $\begin{array}{l}\text { Under/Over } \\
40 \text { at the onset }\end{array}$ & $\begin{array}{l}\text { Disease } \\
\text { Status }\end{array}$ & $\begin{array}{l}\text { BC } \\
\text { Familiarity }\end{array}$ & $\begin{array}{l}\text { BRCA1/2 } \\
\text { Mutational } \\
\text { status * }\end{array}$ & $\begin{array}{l}\text { Tissue } \\
\text { Histology }\end{array}$ & $\begin{array}{l}\text { Menarche } \\
\text { Age }\end{array}$ & Pregnancies & $\begin{array}{l}\text { Other } \\
\text { Features }\end{array}$ \\
\hline P1 & OVER & breast cancer & no & Wt & $\mathrm{nr}$ & $\mathrm{nr}$ & $\mathrm{nr}$ & $\mathrm{nr}$ \\
\hline P2 & UNDER & breast cancer & yes & Wt & luminal A & 12 & 1 & oral contraceptives \\
\hline P3 & OVER & $\angle A B C$ & yes & $W t$ & Her2 related & 13 & 0 & oral contraceptives \\
\hline P4 & OVER & $\angle A B C$ & no & Wt & TNBC & 11 & 3 & smoke \\
\hline P5 & UNDER & breast cancer & yes & Wt & luminal A & 11 & 2 & $\mathrm{nr}$ \\
\hline P6 & OVER & breast cancer & yes & Wt & luminal A & 11 & 1 & smoke, obesity, ovarian stimulation \\
\hline P7 & OVER & breast cancer & yes & Wt & $\mathrm{nr}$ & 11 & 3 & smoke, oral contraceptives \\
\hline P8 & UNDER & $\mathrm{LABC}$ & yes & Wt & TNBC & 14 & 3 & smoke \\
\hline P9 & UNDER & breast cancer & Yes & BRCA1 & TNBC & 12 & 3 & $\mathrm{nr}$ \\
\hline P10 & UNDER & breast cancer & yes & Wt & luminal A & 9 & 0 & oral contraceptives \\
\hline P11 & UNDER & breast cancer & no & Wt & $\mathrm{nr}$ & 11 & 2 & oral contraceptives \\
\hline P12 & UNDER & breast cancer & yes & Wt & luminal A & 9 & 1 & $\mathrm{nr}$ \\
\hline P13 & OVER & breast cancer & yes & Wt & $\mathrm{nr}$ & 11 & 1 & ovarian stimulation \\
\hline P14 & OVER & $\mathrm{LABC}$ & yes & Wt & $\mathrm{nr}$ & 14 & 3 & $\mathrm{nr}$ \\
\hline P15 & UNDER & breast cancer & Yes & BRCA2 & luminal A & 16 & 1 & oral contraceptives, smoke \\
\hline P16 & UNDER & breast cancer & yes & Wt & luminal A & 10 & 3 & $\mathrm{nr}$ \\
\hline P17 & UNDER & breast cancer & no & Wt & luminal A & 12 & 2 & oral contraceptives, smoke \\
\hline P18 & UNDER & breast cancer & no & Wt & Her2 related & 11 & 0 & smoke \\
\hline P19 & OVER & LABC & yes & Wt & luminal A & 14 & $\mathrm{nr}$ & $\mathrm{nr}$ \\
\hline P20 & UNDER & breast cancer & no & Wt & luminal B & 12 & 1 & oral contraceptives \\
\hline P21 & UNDER & breast cancer & yes & Wt & luminal B & 13 & 0 & oral contraceptives, smoke \\
\hline P22 & OVER & $\mathrm{LABC}$ & yes & Wt & $\mathrm{nr}$ & 12 & 2 & smoke \\
\hline P23 & UNDER & breast cancer & yes & Wt & $\mathrm{nr}$ & 13 & 2 & $\mathrm{nr}$ \\
\hline P24 & OVER & breast cancer & yes & Wt & $\mathrm{nr}$ & 11 & 2 (1 abort.) & $\mathrm{nr}$ \\
\hline P25 & UNDER & breast cancer & no & Wt & luminal B & 16 & 1 & oral contraceptives, smoke \\
\hline P26 & OVER & $\mathrm{LABC}$ & yes & Wt & luminal A & 14 & 2 & $\mathrm{nr}$ \\
\hline P27 & UNDER & breast cancer & Yes & BRCA2 & luminal B & 13 & 2 & oral contraceptives \\
\hline P28 & UNDER & breast cancer & yes & Wt & $\mathrm{nr}$ & 13 & 0 & $\mathrm{nr}$ \\
\hline P29 & UNDER & breast cancer & no & Wt & luminal B & 13 & $\mathrm{nr}$ & $\mathrm{nr}$ \\
\hline P30 & UNDER & breast cancer & yes & Wt & Her2 related & 12 & 1 & oral contraceptives \\
\hline P31 & UNDER & breast cancer & yes & Wt & $\mathrm{nr}$ & 12 & 2 & oral contraceptives \\
\hline P32 & UNDER & breast cancer & no & $W t$ & $\mathrm{nr}$ & 12 & 6 & smoke \\
\hline P33 & UNDER & breast cancer & yes & Wt & luminal B & 14 & 2 & ovarian stimulation \\
\hline P34 & UNDER & breast cancer & no & Wt & luminal B & 13 & 1 & $\mathrm{nr}$ \\
\hline
\end{tabular}

LABC is locally advanced breast cancer ( $n=7$ patients); TNBC is triple negative breast cancer; BRCA mutated patients $(n=3) ; n r:$ not reported

Table 2 Primers used to amplify the V4-V6 regions encoding for the $16 \mathrm{~S}$ rRNA for sequencing library preparations

\begin{tabular}{ll}
\hline ID of the 16 S primer & Sequence \\
\hline Forward 16 SV4-V6 & TCGTCGGCAGCGTCA \\
& GATGTGTATAAGAGA \\
& CAGCAGCAGCCGCG \\
& TAATAC \\
Reverse 16SV4-V6 & GTCTCGTGGGCTCGG \\
& AGATGTGTATAAGAG \\
& ACAGTGACGACAG \\
& CCATGC \\
\hline
\end{tabular}

Illumina 16 S PCR primers with overhang adapters and sequences complementary to $\mathrm{V} 4-\mathrm{V} 6$ regions (in bold) through the Illumina MiSeq System (PE $300 \times 2$ ), by obtaining an average read length of about $300 \mathrm{bp}$. The raw sequencing data are available in the SRA repository under the BioProject PRJNA759366.

\section{Bioinformatic Analysis and Statistics}

The Illumina MiSeq paired-end (PE) reads were denoised using a procedure relying on the inference of the Amplicon Sequence Variants (ASVs) (i.e. an estimation of the actual amplicons). The PE reads were treated with cut adapt to remove Illumina adaptors [26]. The trimmed reads were merged using PEAR [27]. The resulting 
merged reads were denoised by applying the DADA2 workflow [28]. This procedure included the chimera(i.e. PCR artifacts) and PhiX- (i.e. the PhiX phage is used during Illumina library preparation to increase nucleotide variability) removal [29-31]. ASV sequences were mapped against the human genome (release hg19) using bowtie 2 to remove nonspecific amplification products (i.e. $16 \mathrm{~S}$ rRNA mitochondrial gene) [32].

The ASVs obtained were taxonomically annotated in BioMaS using the Ribosomal (RDP) database (release $11.5)$ and the NCBI taxonomy as $16 \mathrm{~S}$ rRNA reference collection and taxonomy, respectively [33-36]. In particular, the query sequences were aligned to the reference collection using bowtie2, and the resulting alignments were filtered according to query coverage $(\geq 70 \%)$ and identity percentage $(\geq 90 \%)$. A phylogenetic tree was inferred using the QIIME2 align-to-tree-mafft-fasttree plugin: a multiple sequence alignment of ASV sequences was obtained by using MAFFT and the phylogenetic tree was inferred by applying the maximum-likelihood procedure implemented in Fasttree 2 [37-39].

The taxonomic classification was performed using TANGO [40]. In particular, for ASV sequences obtaining matches with an identity percentage equal or higher than $97 \%$ the classification at species level was accepted, otherwise ASVs were classified at higher taxonomic ranks [41]. The ASV table was normalized by using rarefaction for diversity analysis [42]. The Shannon and the Faith Phylogenetic indices [43, 44] were inferred as alpha diversity measure by applying the phyloseq R-package, and statistically relevant differences between groups were evaluated by applying the Wilcoxon test [45]. The principal coordinates analysis (PCoA) that describes the diversity between the samples (i.e. Beta-diversity) based on the weighted and unweighted UNIFRAC metrics, were inferred by using the vegan $\mathrm{R}$ package and evaluated by PERMANOVA [46, 47].

The statistical comparison between the healthy and tumor samples was performed by using DESeq2 [48]. To measure differences between tissues in the different conditions, the data were normalized by taking into account inter-sample variability. The $\mathrm{p}$-values obtained were adjusted for multiple comparisons with the Benjamin-Hochberg method. Finally, a supervised model for sample classification was built using the Random Forest (RF) Machine Learning (ML) methods and the R package caret [49]. In particular, the DESeq2 ASVs normalized counts were scaled and centered. Then the dataset was randomly divided into the training set and the test data set that including 54 and 14 samples, respectively. The tuning of RF hyperparameter mtry was performed by repeating cross-validation ( 10 cross-validation with 10 repeats) on the training dataset and the best mtry value was selected according to ROC metric. Lastly, the accuracy of the RF model was assessed on the test dataset.

\section{Results}

The comparison between the breast tissue microbiota in tumor and that in paired normal adjacent tissues from 34 women affected by breast cancer enabled us to investigate the distribution of microbial communities of each sample. Each sample obtained more than $90 \%$ of reads thereby passing quality filtering with an average quality value of $30(\mathrm{Q} 30)>80 \%$. The analyzed data were produced by performing an Illumina MiSeq sequencing run, and we obtained a variable number of Paired End (PE) reads per sample (mean 130,820, sd 384,926.925, median $69,920$, min 13,417 , $\max 3,215,914)$. About $96 \%$ of input sequences passed the trimming of adaptors and the PCR primer step.

The overall quality of reverse reads was lower than that of forward reads for all the sequenced samples and, in this specific case, did not pass the quality filter in dada2 [50]. To overcome this issue, we applied an approach based on PE reads merging before denoising $[51,52]$. About $70 \%$ of input reads were successfully merged. The denoising step enabled us to infer the Amplicon Sequence Variants (ASV) sequences and their absolute counts. The ASV sequences were checked to remove chimeras and human contaminants. In order to achieve an adequate compromise between the microbiome sampling and the number of retained samples, the ASV table was rarefied using an equal sequencing depth of 15,000 (Additional File 1: Figure S1), 27 and 16 tumoral and non-tumoral samples were retained, respectively.

The alpha diversity was measured using the Shannon Index and plotted as a box-plot (Fig. 1a). No statistically significant differences were observed between the tested conditions according to the Shannon index (p-value = 0.1649). Conversely, the distribution of the Faith index differed significantly ( $p$-value $\leq 0.05$ ) between healthy and tumor tissue samples (Fig. 1b).

Although no clear clustering was observed in the PCoA plot based on weighted UniFrac analysis (Fig. 1c) between healthy $(\mathrm{H})$ and tumor $(\mathrm{T})$ tissue samples, the PERMANOVA suggested that about $7 \%$ of the observed variability is explained by the conditions ( $\mathrm{p}$-value $=$ 0.007). Conversely, neither the PCoA plots nor the PERMANOVA based on unweighted UniFrac (data not reported) resulted in any significant difference between the two conditions ( $\mathrm{p}$-value $=0.103$ ).

\section{Taxonomic Distribution}

All the ASVs were taxonomically annotated at least at kingdom level. Generally, 13 phyla, 25 classes, 59 orders, 105 families, 199 genera and 514 species were 

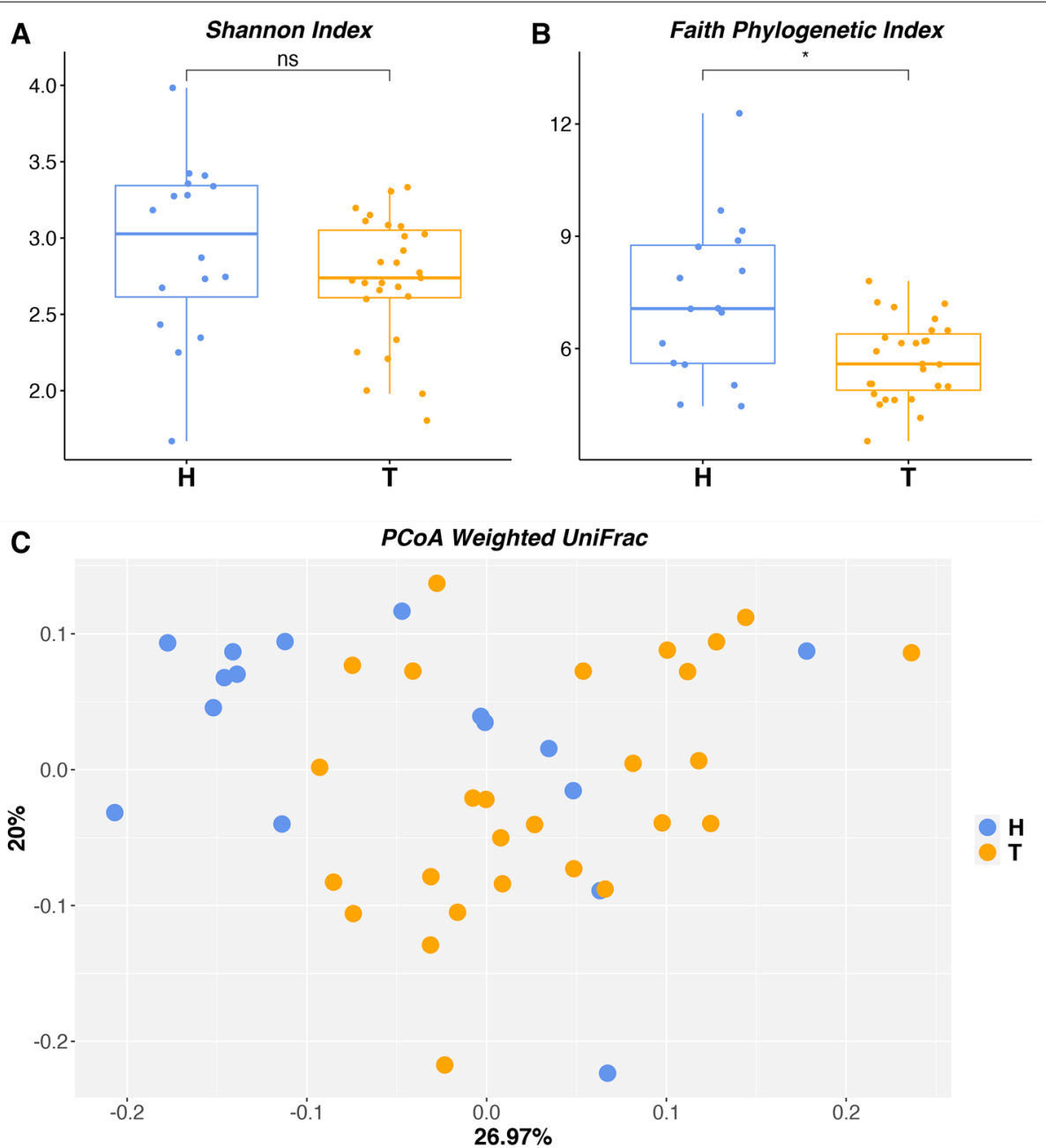

Fig. 1 A. The distribution of the inferred Shannon Index for tumoral and non-tumoral samples were shown as boxplot. B. The distribution of the inferred Faith Phylogenetic Index for tumoral and non-tumoral samples were shown as boxplot. C. PCoA plot based on weighted UniFrac measurements. H: healthy tissue; T: tumor tissue

identified across all samples. The distribution of phyla is shown in Fig. 2. The most predominant phyla are Actinobacteria and Proteobacteria (about $31 \%$ and $55.4 \%$ on average, respectively). Gammaproteobacteria (40.22\%), Actinobacteria (25.09\%), Bacilli (7.83\%) and Alphaproteobacteria (5.57\%) are the most abundant classes among all tumor and normal samples.

The most prevalent families are Propionibacteriaceae (23.57\%), Moraxellaceae (17.83\%) and Pseudomonadaceae (15.19\%). The genera Propionibacterium (22.59\%), Acinetobacter (15.43\%) and Pseudomonas $(15.10 \%)$ are the most abundant. The results of statistical comparisons are reported in Table 3. The box-plot of each statistically different taxon between healthy and tumor samples, are shown in Fig. 3 (A-F) and Fig. 4 (A-C), and in Additional File 1: Figure S2. Overall, in non-tumoral paired samples a higher abundance of taxa belonging the Actinobacteria phylum was found. In particular, the order Propionibacteriales, the family Propionibacteriaceae, the genus Propionibacterium and species Propionibacterium sp. enrichment culture clone MRHull-FeSM-11R and Propionibacterium acnes are more abundant in non-tumoral tissues (Fig. $3 \mathrm{~A}-\mathrm{F}$ and Fig. $4 \mathrm{C}$ ). Conversely, Firmicutes and Alpha-proteobacteria are significantly overrepresented in tumoral tissues.

In order to identify the ASVs able to discern among tumoral and non-tumoral tissues by using a robust and reliable method, a supervised classification machine learning model was built using Random Forest (RF). To avoid overfitting and to properly train the model, the dataset was divided into a training and a test dataset, accounting for 54 and 14 samples, respectively. 


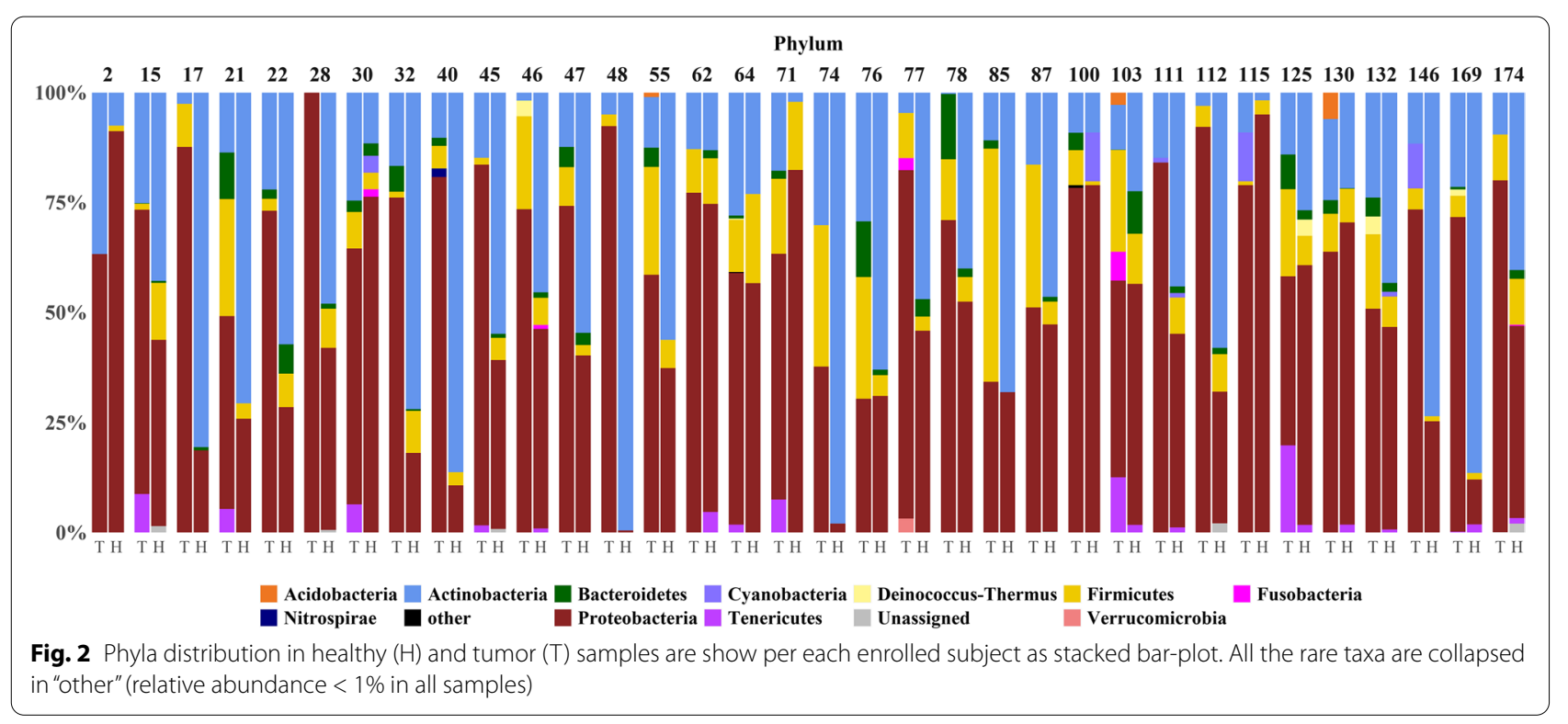

Table 3 List of taxa that differ significantly between healthy and tumor samples

\begin{tabular}{|c|c|c|c|}
\hline Taxa & Significance & Log2 fold change & Adjusted $p$-value \\
\hline \multicolumn{4}{|l|}{ Phylum } \\
\hline Actinobacteria & $* * * *$ & 2.28 & $1.36 \mathrm{e}^{-11}$ \\
\hline Firmicutes & $*$ & -0.89 & 0.047 \\
\hline \multicolumn{4}{|l|}{ Class } \\
\hline Actinobacteria (Class) & $* * * *$ & 2.51 & $3.23 \mathrm{e}^{-12}$ \\
\hline Alphaproteobacteria & * & -1.51 & 0.015 \\
\hline \multicolumn{4}{|l|}{ Order } \\
\hline Propionibacteriales & $* * * *$ & 2.53 & $2.06 \mathrm{e}^{-11}$ \\
\hline Aeromonadales & $* * * *$ & 26.23 & $1.99 e^{-19}$ \\
\hline Selenomonadales & $* * * *$ & 25.6 & $2.17 e^{-18}$ \\
\hline \multicolumn{4}{|l|}{ Family } \\
\hline Propionibacteriaceae & $* * * *$ & 2.54 & $7.24 e^{-08}$ \\
\hline Aeromonadaceae & $* * * *$ & 25.18 & $4.60 e^{-21}$ \\
\hline \multicolumn{4}{|l|}{ Genus } \\
\hline Propionibacterium & $* * * *$ & 2.36 & $3.39 e^{-06}$ \\
\hline Aeromonas & $* * * *$ & 26.32 & $1.00 e^{-19}$ \\
\hline \multicolumn{4}{|l|}{ Species } \\
\hline Variovorax sp. WO3 & $* * * *$ & 25.13 & $1.28 \mathrm{e}^{-18}$ \\
\hline Moraxella sp. S2 & $* * * *$ & -25.4 & $1.42 \mathrm{e}^{-17}$ \\
\hline Pseudomonas sp. PS9 (2007) & $* * * *$ & 25.14 & $4.39 e^{-21}$ \\
\hline $\begin{array}{l}\text { Propionibacterium sp. } \\
\text { Enrichment culture clone MRHull-FeSM-11E }\end{array}$ & $* * *$ & 27.3 & $2.28 e^{-35}$ \\
\hline Pseudomonas sp. IMER-A2-21 & $* * * *$ & 26.39 & $9.32 e^{-22}$ \\
\hline Pseudomonas brenneri & $* * * *$ & -25.87 & $4.13 e^{-18}$ \\
\hline Neisseria elongata & $* * * *$ & 25.00 & $4.22 e^{-17}$ \\
\hline Propionibacterium acnes & $* * *$ & 1.91 & 0.006 \\
\hline
\end{tabular}

The analysis was performed by comparing healthy and tumor samples, consequently if the log2 fold change is positive, the taxon counts are higher in healthy than in tumor samples. Significance level refers to adjusted p-values: ${ }^{*} \leq 0.05,{ }^{* *} \leq 0.01,{ }^{* * *} \leq 0.001,{ }^{* * * *} \leq 0.0001$ 


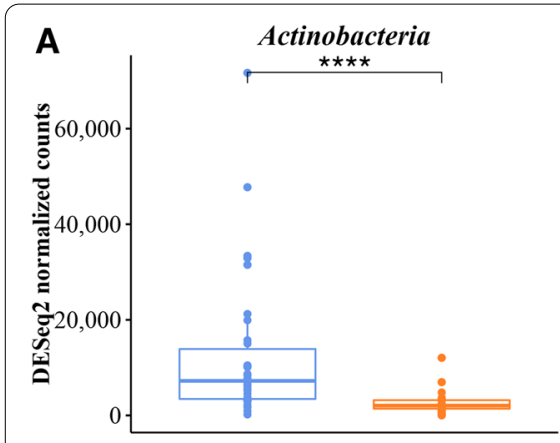

审 $\mathbf{H}$ 舟 $\mathbf{T}$

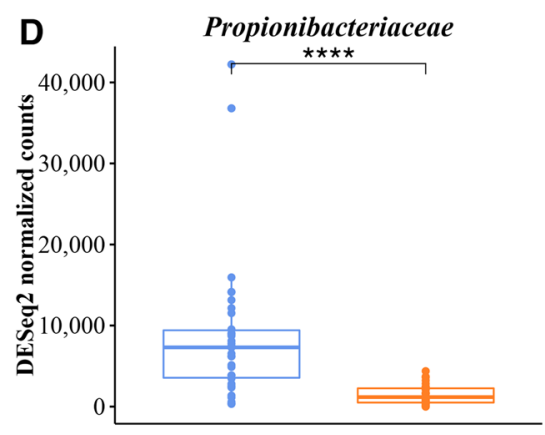

审 $\mathbf{H}$ 察 $\mathbf{T}$

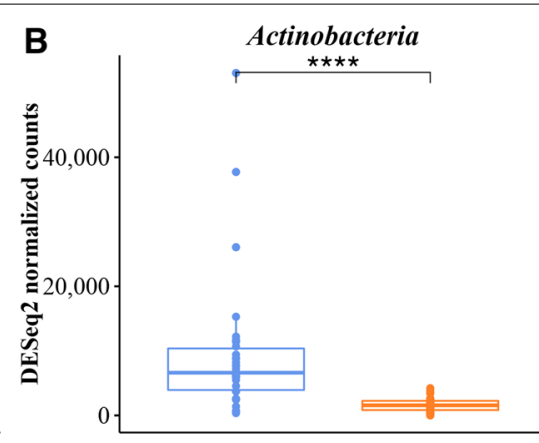

审 $\mathbf{H}$ 䁇 $\mathbf{T}$

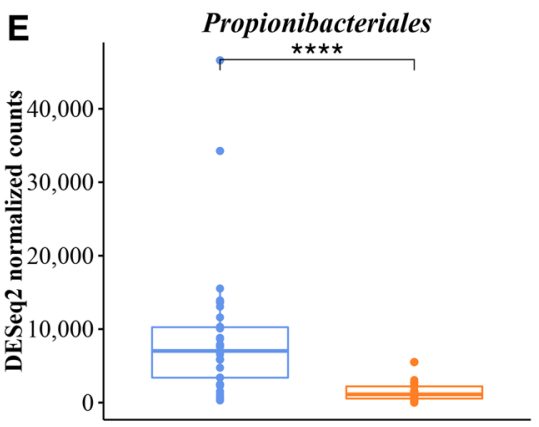

察 $\mathbf{H}$ 亩 $\mathbf{T}$

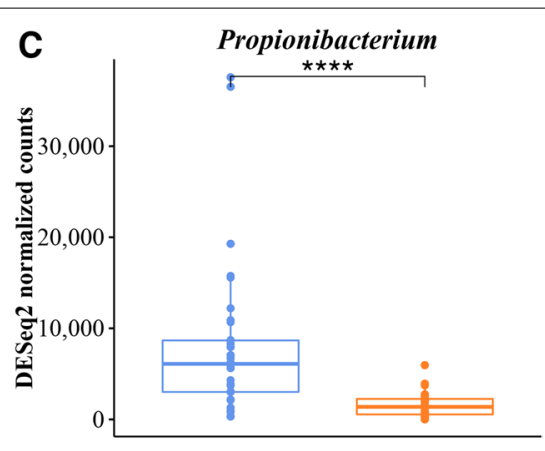

审 $\mathbf{H}$ 自 $\mathbf{T}$

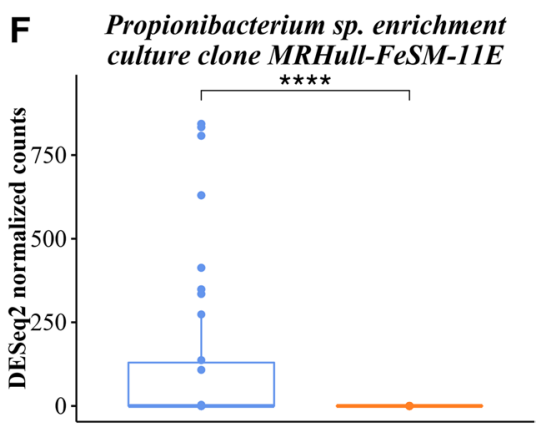

审 $\mathbf{H}$ 察 $\mathbf{T}$

Fig. 3 (panels A-F). Normalized read counts distribution of statistically different taxa between healthy and tumor samples were shown as box-plot. In detail, Actinobacteria at phylum level, p-value=1,36E-11; Actinobacteria at class level, p-value=3,23E-12; Propionibacterium at genus level, $\mathrm{p}$-value =3,39E-06; Propionibacteriaceae at family level, $\mathrm{p}$-value =7,24E-08; Propionibacteriales at order level, $\mathrm{p}$-value=2,06E-11; Propionibacterium_ sp._enrichment_culture_clone_MRHull-FeSM-11, at species level, $\mathrm{p}$-value=2,28E-35. (Other box plots are shown in Additional File 1, Figure S1)

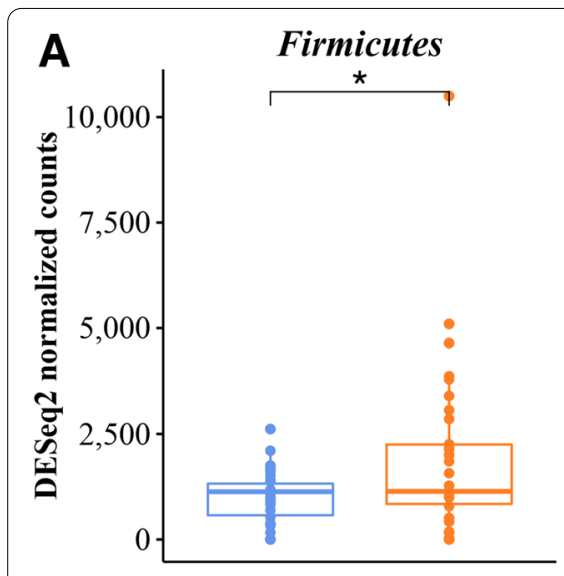

审 $\mathbf{H}$ 客 $\mathbf{T}$

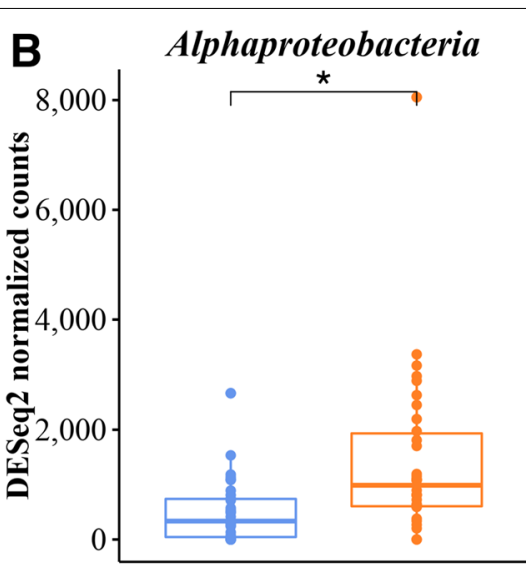

家 $\mathbf{H}$ 宛

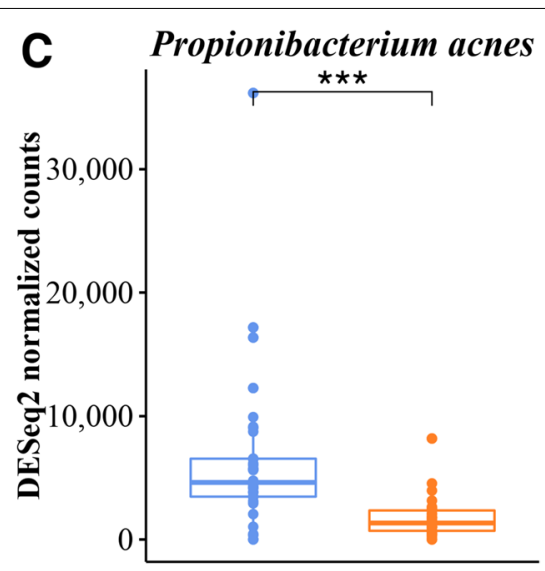

H审 $\mathbf{T}$

Fig. 4 (Panels A-C). Normalized read counts distribution of statistically different taxa between healthy and tumor samples were shown as box-plot. Firmicutes at phylum level, $p$-value $=0,047230491$; Alphaproteobacteria at class level, $p$-value $=0.014872974$; Propionibacterium_acnes at species level, $p$-value $=0,0005835$ 


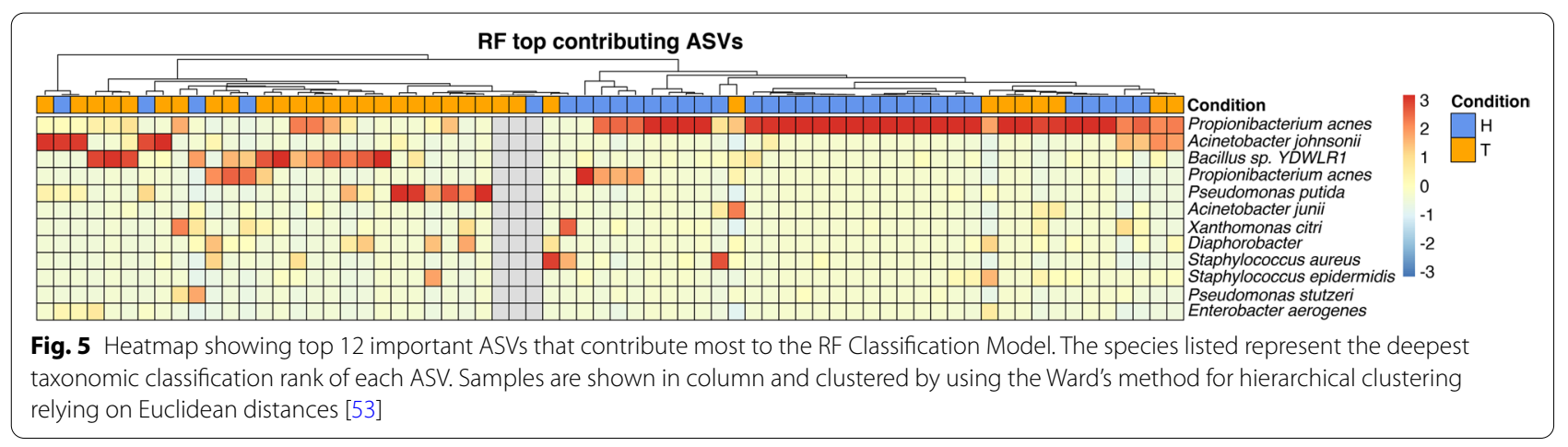

The overall accuracy of the test dataset was about $89 \%$, with two misclassifications for healthy samples. The ASVs that most contribute to the model accuracy were selected and used to plot a heatmap (Fig. 5).

As shown in Fig. 5, two main clusters may be identified, the first one is constituted mainly by healthy tissues and the second one by tumor tissues. The first ASV was assigned to Propionibacterium acnes and was principally observed in healthy tissues. This result agrees with those obtained by comparing taxa abundances in DESeq2.

Regarding BRCA mutational status, there were only three BRCA-positive patients in our population and in particular one carrying a mutation in the $B R C A 1$ gene and two in BRCA2 gene. Consequently, the data were not enough to carry a reliable statistical analysis. Similarly, the same issue was observed for other confounding factors, i.e. smoking status and contraception usage.

\section{Discussion}

Studies of the entire microbial communities and their relationships with the host have been conducted to evaluate how their imbalance could be involved in health maintaining and diseases [20, 54-61]. In particular, several studies have linked the microbiome to the initiation and progression of different types of cancer, including breast cancer $[58,59]$. Moreover, the cooperation of microbial communities' imbalance with diet, obesity, estrogens and immune modulation has been considered an important promoter of breast cancer [12, 62]. Notably, the majority of authors $[16-19,24,36,37,61-64]$ note that their findings are hypothesis-generators and support further investigations to identify a microbial risk signature for breast cancer and potential microbial-based prevention and/or therapies.

In this scenario, we studied the resident breast microbiota in tumor and paired normal breast tissue from $34 \mathrm{BC}$ patients. The aim of our study was to evaluate the microbial composition of breast tumor tissues and healthy tissues in the attempt to shed light on the link between dysbiosis and breast cancer which, in turn, may indicate that a change in bacterial species could contribute to the modulation of cancer development. A comparison between paired healthy and tumor tissues revealed differences of bacterial community and composition. The number of ASVs detected between paired normal and tumor tissue showed significant differences in richness between the sampled communities. Proteobacteria and Actinobacteria showed differences between two groups: healthy tissues showed an increase of Actinobacteria and a decrease of Proteobacteria; the opposite appeared in tumor tissues. Conversely, in healthy tissues, appear to be more prevalent Propionibacterium and Pseudomonas.

In particular, we observed an overall decrease of microbial alpha diversity in tumoral tissues compared to healthy ones. We also found a significant depletion of Propionibacterium acnes in tumor tissues versus normal breast tissues, which is a novel finding. Propionibacterium acnes (currently denominated Cutibacterium acnes) is a component of the human microbiome found in several body districts. Its over-representation in normal tissue was observed by comparing abundances (DESeq2) and also by machine learning (Random Forest), which indicates that these results are robust. This gram-positive species is considered an opportunist pathogen because potentially pathogenic genes were found in the genomes available (5 phylotypes). However, the role of Propionibacterium remains to be established. For example, Talib et al. 2015 [65] described a potential antitumoral action of Propionibacterium acnes in breast cancer, and Portillo et al. 2013 [66] suggested that it plays a role in implantassociated infections.

Our study supports the presence of microbial DNA in breast tissues that could probably influence the local tissue microenvironment. In the attempt to minimize any external variations (including sample preparation and sequencing) between healthy and tumor tissues, we compared healthy tissues to the paired tumor breast tissues taken from each woman at the same time and in the same 
conditions. All 68 samples were amplified, purified and sequenced together in a single sequencing run in order to minimize any analytical variation. Although, our results are at variance from those reported by others [16-19, $24,61,62]$, it is important to highlight that differences in both experimental procedures (i.e., primer design and the use of bioinformatic pipelines to filter and to analyze data) and different cohort enrolled can affect results and, therefore, their comparison. Survey results about the breast cancer tissue microbiome, are reported for a more comprehensive comparison (Additional File 1: Table S1) and it is important to note how several factors, such as ethnicity, dietary habits, geographical origin, lactation status, pharmaco-therapeutic before surgery, the method of sample collection $[66,67]$ can affect the composition of microbial tissues [16]. For instance, Fusobacterium nucleatum has been described as a key player in several pathological conditions, and particularly in colon rectal cancer. However, earlier work was principally based on a comparison between healthy and unhealthy samples [16$19,24]$, not including paired tissues analysis.

Accordingly, another key difference is that the primer pairs we used differed from those used in other studies. In their review of the association between the gut/breast microbiota and breast cancer, Laborda-Illanes et al. 2020 [20] highlighted the differences among studies in terms of data results. We counted 6 different combinations of the $16 \mathrm{~S}$ hypervariable region in 10 papers (i.e., $\mathrm{V} 4=3$, $\mathrm{V} 6=2, \mathrm{~V} 3-\mathrm{V} 4=1, \mathrm{~V} 3-\mathrm{V} 5=2, \mathrm{~V} 1-\mathrm{V} 2=1$ and $\mathrm{V} 3=1)$. Consequently, it may be misleading to compare surveys conducted using different marker regions, also considering the different efficiency in target amplification and in the resolution of taxonomic assignment.

Therefore, it is difficult and also controversial to define a precise signature of the breast cancer microbiome. Thus, our effort was not to define a universal bacterial signature in breast cancer tissues, but to reinforce the concept that it is an altered balance that characterizes tumor tissues versus healthy tissues in the same woman, also at the very close proximity regions, which per se increases significance of the microbial presence at the level of breast tissue cell transformation. Indeed, we also found that microbial alpha diversity was overall lower in tumor tissues than in healthy tissues.

Larger studies, conducted in diverse geographic regions, are needed to define - if existing - a precise bacterial signature for each type of tissue neoplasia and thus to determine the role played by the microbiome in breast cancer onset and development. Furthermore, it is difficult to use general approaches in different cohorts particularly those living in different geographic regions. Rather, it may be more effective to study patients, cohortby-cohort or groups of subjects living in the same region and under similar environmental conditions. It is also necessary to understand, using in vitro systems as human tumoroids and mouse models, how different pre-surgery antibiotic regimens can induce disturbances in the breast microbiota and how these disturbances affect $\mathrm{BC}$ progression. Indeed, the lack of this information may represent a limitation.

It is now necessary to understand the effect that the metabolites produced from resident bacteria have on the development and progression of the breast. However, it is necessary not only to study the association among microbiota, tumor development and progression and/or anti-tumor immune responses using metagenomic sequencing technologies, but also to demonstrate microbiota functionality using transcriptional and/or metabolic profiling $[68,69]$, thereby paving the way to the application of further precision medicine in $\mathrm{BC}$ patients.

\section{Conclusions}

This study reveals a highly significant difference in the abundance of the various taxa of the microbiome in breast tumor tissues versus their healthy tumor-adjacent counterparts in women after surgery. These alterations reflect qualitative and quantitative differences of taxa, thus indicating their relevance in the comprehension of microbiome content and their role in tumor tissues.

Finally, assessing the different microbial composition in relation to $\mathrm{BC}$ onset and progression could be a goal to achieve in future studies on more numerous cohorts of patients.

\section{Abbreviations \\ ASVs: AmpliconSequence Variants; BC: breastcancer; ER: estrogenreceptor; ML: MachineLearning; NGS: nextgeneration sequencing; PE: pairedend; RF:} RandomForest.

\section{Supplementary Information}

The online version contains supplementary material available at https://doi. org/10.1186/s12885-021-09074-y.

Additional file 1: Figure S1. Rarefaction curves used to define the rarefaction threshold. Figure $\mathbf{S} 1$. shows box-plot of statistically different taxa between healthy and tumor samples. Table S1. lists the differences among data obtained in different cohorts of patients in several studies by different Authors for results comparison.

\section{Acknowledgements}

We thank Drs Ilaria Granata and Mario Guarracino for their helpful suggestions and discussions during bioinformatic data analysis. We thank Jean Ann Gilder for text editing, with special reference to English language.

\section{Authors' contributions}

Conceptualization, F.S.; methodology, F.S., M.V.E., B.F.; bioinformatics and statistical analyses, G.P., B.F.; supervision, validation of all bioinformatic analysis and statistical process, G.P., B.F.; wet lab experiments M.V.E., M.N., V.D.; first bioinformatics approaches, G.C.; resources, F.S.; data curation, F.S., G.P., B.F.; writing original draft preparation, F.S., M.V.E., B.F., M.N.; writing review and editing, 
F.S., G.P.; visualization and supervision, M.V.E, M.N., F.S., G.P. and B.F.; projects administration and funding acquisition, F.S; histopathology experiments and tissue surgical availability G.B., M.D. and A.C. All authors have contributed to read and agreed to this published version of the manuscript.

\section{Funding}

This work, in particular the wet experimental phase, was supported by grants PON03PE_00060_2 and PON03PE_00060_7 (Campania - Bioscience) from the Italian Ministry of University and Research (to F.S.), and CIRO and SATIN grants (to F.S.) from Regional (Campania Region, Italy) funds, including 2017, 2020 and 2021 Campania Region contribution.

\section{Availability of data and materials}

The raw sequencing data are available in the Sequence Read Archive (SRA) repository under the BioProject PRJNA759366.

\section{Declarations}

\section{Ethics approval and consent to participate}

The study was conducted according to the guidelines of the Declaration of Helsinki, and approved by the Istituto Nazionale Tumori - Fondazione G. Pascale Ethics Committee (protocol number 3 of 03/25/2009). Informed Consent Statement: Written Informed consent was obtained from all subjects involved in the study.

\section{Consent for publication}

Not applicable.

\section{Competing interests}

The authors declare that they have no competing interests.

\section{Author details}

${ }^{1}$ Department of Molecular Medicine and Medical Biotechnologies, University Federico II, Via Sergio Pansini, 5, 80131 Napoli, NA, Italy. ${ }^{2}$ CEINGE - Biotecnologie Avanzate, Via Gaetano Salvatore, 486, 80145 Napoli, Italy. ${ }^{3}$ Institute of Biomembranes, Bioenergetics and Molecular Biotechnologies, Consiglio Nazionale delle Ricerche, Via Giovanni Amendola, 122/O, 70126 Bari, BA, Italy. ${ }^{4}$ Evolve Biosystems, Inc, 95618 Davis, CA, USA. ${ }^{5}$ Department of Human Sciences and Quality of Life Promotion, San Raffaele Open University, Via di Val Cannuta, 247, 00166 Rome, Italy. ${ }^{6}$ Department of Senology, Istituto Nazionale Tumori - IRCCS, 'Fondazione Pascale', Via Mariano Semmola, 53, 80131 Napoli, NA, Italy. ${ }^{7}$ Clinica Villa Fiorita, Via Filippo Saporito, 24, 81031 Aversa, CE, Italy. ${ }^{8}$ Scientific Directorate, Istituto Nazionale Tumori, Fondazione G. Pascale, IRCCS, Via Mariano Semmola, 53, 80131 Napoli, NA, Italy. ${ }^{9}$ Department of Biosciences, Biotechnology and Biopharmaceutics, University of Bari "A. Moro", Piazza Umberto I, 1, BA 70121 Bari, Italy.

Received: 12 July 2021 Accepted: 30 November 2021 Published online: 03 January 2022

\section{References}

1. Coughlin SS. Epidemiology of Breast Cancer in Women. Adv Exp Med Biol. 2019;1152:9-29.

2. Van Cott C. Cancer Genetics. Surg Clin North Am. 2020;100:483-98.

3. Armstrong N, Ryder S, Forbes C, Ross J, Quek RGW. A systematic review of the international prevalence of BRCA mutation in breast cancer. Clin Epidemiol. 2019;11:543-61.

4. Stenehjem DD, Telford C, Unni SK, Bauer H, Sainski A, Deka R, et al. BRCA testing and outcomes in women with breast cancer. Breast Cancer Res Treat. 2021;186:839-850.

5. Esposito MV, Nunziato M, Starnone F, Telese A, Calabrese A, D'Aiuto G, et al. A novel pathogenic BRCA1 splicing variant produces partial intron retention in the mature messenger RNA. Int J Mol Sci. 2016;17:2145.

6. Nunziato M, Starnone F, Lombardo B, Pensabene M, Condello C, Verdesca $F$, et al. Fast detection of a BRCA2 large genomic duplication by next generation sequencing as a single procedure: A case report. Int J Mol Sci. 2017;18:2487.
7. Esposito MV, Minopoli G, Esposito L, D’Argenio V, Di Maggio F, Sasso E, et al. A functional analysis of the unclassified Pro2767Ser BRCA2 variant reveals its potential pathogenicity that acts by hampering DNA binding and homology-mediated DNA repair. Cancers (Basel). 2019;1 1:1454.

8. Hu C, Hart SN, Gnanaolivu R, Huang H, Lee KY, Na J, et al. A PopulationBased Study of Genes Previously Implicated in Breast Cancer. N Engl J Med. 2021;384:440-51.

9. Dorling L, Carvalho S, Allen J, González-Neira A, Luccarini C, Wahlström C, et al. Breast Cancer Risk Genes - Association Analysis in More than 113,000 Women. N Engl J Med. 2021;384:428-39.

10. Fanale D, Incorvaia L, Filorizzo C, Bono M, Fiorino A, Calò V, et al. Detection of germline mutations in a cohort of 139 patients with bilateral breast cancer by multi-gene panel testing: Impact of pathogenic variants in other genes beyond brca1/2. Cancers (Basel). 2020;12:2415.

11. Nasir A, Bullo MMH, Ahmed Z, Imtiaz A, Yaqoob E, Jadoon M, et al. Nutrigenomics: Epigenetics and cancer prevention: A comprehensive review. Crit Rev Food Sci Nutr. 2020;60:1375-87.

12. Bodai BI, Nakata TE. Breast Cancer: Lifestyle, the Human Gut Microbiota/ Microbiome, and Survivorship. Perm J. 2020;24:129.

13. Alizadehmohajer N, Shojaeifar S, Nedaeinia R, Esparvarinha M, Mohammadi F, Ferns GA, et al. Association between the microbiota and women's cancers - Cause or consequences? Biomed Pharmacother. 2020;127:11020.

14. Yu Q, Jobin C, Thomas RM. Implications of the microbiome in the development and treatment of pancreatic cancer: Thinking outside of the box by looking inside the gut. Neoplasia (United States). 2021;23:246-56.

15. Komorowski AS, Pezo RC. Untapped "-omics": the microbial metagenome, estrobolome, and their influence on the development of breast cancer and response to treatment. Breast Cancer Res Treat. 2020;179:287-300.

16. Smith A, Pierre JF, Makowski L, Tolley E, Lyn-Cook B, Lu L, et al. Distinct microbial communities that differ by race, stage, or breast-tumor subtype in breast tissues of non-Hispanic Black and non-Hispanic White women. Sci Rep. 2019:9:1-10.

17. Hieken TJ, Chen J, Hoskin TL, Walther-Antonio M, Johnson S, Ramaker $\mathrm{S}$, et al. The microbiome of aseptically collected human breast tissue in benign and malignant disease. Sci Rep. 2016;6:30751.

18. Urbaniak C, Gloor GB, Brackstone M, Scott L, Tangney M, Reida G. The microbiota of breast tissue and its association with breast cancer. Appl Environ Microbiol. 2016;82:5039-48.

19. Wang H, Altemus J, Niazi F, Green H, Calhoun BC, Sturgis C, et al. Breast tissue, oral and urinary microbiomes in breast cancer. Oncotarget. 2017:8:88122-38

20. Laborda-Illanes A, Sanchez-Alcoholado L, Dominguez-Recio ME, Jimenez-Rodriguez B, Lavado R, Comino-Méndez I, et al. Breast and gut microbiota action mechanisms in breast cancer pathogenesis and treatment. Cancers (Basel). 2020;12:2465.

21. Gubert C, Kong G, Renoir T, Hannan AJ. Exercise, diet and stress as modulators of gut microbiota: Implications for neurodegenerative diseases. Neurobiol Dis. 2020;134:104621.

22. Song M, Chan AT, Sun J. Influence of the Gut Microbiome, Diet, and Environment on Risk of Colorectal Cancer. Gastroenterology. 2020;158:322-40.

23. Zhang X, Pan Z. Influence of microbiota on immunity and immunotherapy for gastric and esophageal cancers. Gastroenterol Rep. 2020;8:206-21.

24. Meng S, Chen B, Yang J, Wang J, Zhu D, Meng Q, et al. Study of microbiomes in aseptically collected samples of human breast tissue using needle biopsy and the potential role of in situ tissue microbiomes for promoting malignancy. Front Oncol. 2018;8:318.

25. D'Argenio V, Esposito MV, Telese A, Precone V, Starnone F, Nunziato M, et al. The molecular analysis of BRCA1 and BRCA2: Next-generation sequencing supersedes conventional approaches. Clin Chim Acta. 2015;446:221-5.

26. Martin M. Cutadapt removes adapter sequences from high-throughput sequencing reads. EMBnet.journal. 2011;17:10-2.

27. Zhang J, Kobert K, Flouri T, Stamatakis A. PEAR: A fast and accurate Illumina Paired-End reAd mergeR. Bioinformatics. 2014;30:614-20.

28. Callahan BJ, McMurdie PJ, Rosen MJ, Han AW, Johnson AJA, Holmes SP. DADA2: High-resolution sample inference from Illumina amplicon data. Nat Methods. 2016;13:581-3. 
29. Nagamine CM, Chan K, Lau YF. A PCR artifact: generation of heteroduplexes. Am J Hum Genet. 1989;45:337-339.

30. Kanagawa T. Bias and Artifacts in Multitemplate Polymerase Chain Reactions (PCR). J Biosci Bioeng. 2003;96:317-23.

31. Mukherjee S, Huntemann M, Ivanova N, Kyrpides NC, Pati A. Largescale contamination of microbial isolate genomes by illumina Phix control. Stand Genomic Sci. 2015;10:18.

32. Langmead B, Salzberg SL. Fast gapped-read alignment with Bowtie 2. Nat Methods. 2012;9:357-9.

33. Santamaria M, Fosso B, Consiglio A, De caro G, Grillo G, Licciulli F, et al. Reference databases for taxonomic assignment in metagenomics. Brief Bioinform. 2012;13:682-95.

34. Fosso B, Santamaria M, Marzano M, Alonso-Alemany D, Valiente G, Donvito G, et al. BioMaS: A modular pipeline for Bioinformatic analysis of Metagenomic AmpliconS. BMC Bioinformatics. 2015;16:203.

35. Cole JR, Wang Q, Cardenas E, Fish J, Chai B, Farris RJ, et al. The Ribosomal Database Project: Improved alignments and new tools for rRNA analysis. Nucleic Acids Res. 2009;37:141-5.

36. Cole JR, Wang Q, Fish JA, Chai B, McGarrell DM, Sun Y, et al. Ribosomal Database Project: Data and tools for high throughput rRNA analysis. Nucleic Acids Res. 2014;42:633-42.

37. Bolyen E, Rideout JR, Dillon MR, Bokulich NA, Abnet CC, Al-Ghalith GA et al. Reproducible, interactive, scalable and extensible microbiome data science using QIIME 2. Nat Biotechnol. 2019;37:852-7.

38. Katoh K, Standley DM. MAFFT multiple sequence alignment software version 7: Improvements in performance and usability. Mol Biol Evol. 2013:30:772-80.

39. Price MN, Dehal PS, Arkin AP. FastTree 2 - Approximately maximumlikelihood trees for large alignments. PLoS One. 2010;5:e9490.

40. Alonso-Alemany D, Barré A, Beretta S, Bonizzoni P, Nikolski M, Valiente G. Further steps in TANGO: Improved taxonomic assignment in metagenomics. Bioinformatics. 2014;30:17-23.

41. Mende DR, Sunagawa S, Zeller G, Bork P. Accurate and universal delineation of prokaryotic species. Nat Methods. 2013;10:881-4.

42. Weiss S, Xu ZZ, Peddada S, Amir A, Bittinger K, Gonzalez A, et al. Normalization and microbial differential abundance strategies depend upon data characteristics. Microbiome. 2017;5:27.

43. Pielou EC. Shannon's Formula as a Measure of Specific Diversity: Its Use and Misuse. Am Nat. 1966;100:463-5.

44. Faith DP. Conservation evaluation and phylogenetic diversity. Biol Conserv. 1992;61:1-10.

45. McMurdie PJ, Holmes S. Phyloseq: An R Package for Reproducible Interactive Analysis and Graphics of Microbiome Census Data. PLoS One. 2013;8:e61217.

46. Chang Q, Luan Y, Sun F. Variance adjusted weighted UniFrac: a powerful beta diversity measure for comparing communities based on phylogeny. BMC Bioinformatics. 2011;12:118.

47. Oksanen J, Blanchet FG, Kindt R, Legendre P, Minchin PR, O'Hara RB, et al. Package vegan: Community Ecology Package. R Packag version 23-1. 2013.

48. Love MI, Huber W, Anders S. Moderated estimation of fold change and dispersion for RNA-seq data with DESeq2. Genome Biol. 2014;15:550.

49. Kuhn M. Building predictive models in R using the caret package. J Stat Softw. 2008;28:1-26.

50. Kwon S, Park S, Lee B, Yoon S. In-depth analysis of interrelation between quality scores and real errors in illumina reads. Proc Annu Int Conf IEEE Eng Med Biol Soc EMBS. 2013;2013:635-8.

51. Barone M, Mendozzi L, D'Amico F, Saresella M, Rampelli S, et al. Influence of a High-Impact Multidimensional Rehabilitation Program on the Gut Microbiota of Patients with Multiple Sclerosis. Int J Mol Sci. 2021;22:7173.

52. Bazzocchi G, Turroni S, Bulzamini MC, D'Amico F, Bava A, et al. Changes in gut microbiota in the acute phase after spinal cord injury correlate with severity of the lesion. Sci Rep. 2021;11:12743.

53. Murtagh F, Legendre P. Ward's Hierarchical Agglomerative Clustering Method: Which Algorithms Implement Ward's Criterion? Journal of Classification. 2014; 31:274-295.

54. Kordy K, Gaufin T, Mwangi M, Li F, Cerini C, Lee DJ, et al. Contributions to human breast milk microbiome and enteromammary transfer of Bifidobacterium breve. PLoS One. 2020;28;15:e0219633.
55. Mostafaei S, Kazemnejad A, Norooznezhad AH, Mahaki B, Moghoofei M. Simultaneous effects of viral factors of human papilloma virus and epstein-barr virus on progression of breast and thyroid cancers: Application of structural equation modeling. Asian Pacific J Cancer Prev. 2020;21:1431-9.

56. Dong TS, Jacobs JP, Hussain SK. Microbial Profiles of Cirrhosis in the Human Small Intestine. Current Gastroenterology Reports. 2019;21:50.

57. Acharya C, Bajaj JS. Gut Microbiota and Complications of Liver Disease. Gastroenterol Clin North Am. 2017;46:155-69.

58. Giuffrè M, Campigotto M, Campisciano G, Comar M, Crocè LS. A story of liver and gut microbes: How does the intestinal flora affect liver disease? A review of the literature. Am J Physiol - Gastrointest Liver Physiol. 2020;318:G889-906.

59. Fan Y, Pedersen O. Gut microbiota in human metabolic health and disease. Nat Rev Microbiol. 2021;19:55-71.

60. Ghosn B, Benisi-Kohansal S, Ebrahimpour-Koujan S, Azadbakht L, Esmaillzadeh A. Association between healthy lifestyle score and breast cancer. Nutr J. 2020;19:4.

61. Veiga P, Pons N, Agrawal A, Oozeer R, Guyonnet D, Brazeilles R, et al. Changes of the human gut microbiome induced by a fermented milk product. Sci Rep. 2014;4:6328.

62. Fernández L, Pannaraj PS, Rautava S, Rodríguez JM. The Microbiota of the Human Mammary Ecosystem. Front Cell Infect Microbiol. 2020;10:586667.

63. Xuan C, Shamonki JM, Chung A, DiNome ML, Chung M, Sieling PA, et al. Microbial dysbiosis is associated with human breast cancer. PLoS One. 2014:9:e83744

64. Costantini L, Magno S, Albanese D, Donati C, Molinari R, Filippone A, et al. Characterization of human breast tissue microbiota from core needle biopsies through the analysis of multi hypervariable 16S-rRNA gene regions. Sci Rep. 2018;8:4-12.

65. Talib WH, Saleh S. Propionibacterium acnes augments antitumor, antiangiogenesis and immunomodulatory effects of melatonin on breast cancer implanted in mice. PLoS One. 2015;10:1-13.

66. Portillo ME, Corvec S, Borens O, Trampuz A. Propionibacterium acnes: An underestimated pathogen in implant-associated infections. Biomed Res Int. 2013;2013:804391.

67. Thyagarajan S, Zhang Y, Thapa S, Allen MS, Phillips N, Chaudhary P, et al Comparative analysis of racial differences in breast tumor microbiome. Sci Rep. 2020;10:14116.

68. Chen RY, Mostafa I, Hibberd MC, Das S, Mahfuz M, Naila NN, et al. A Microbiota-Directed Food Intervention for Undernourished Children. N Engl J Med. 2021;384:1517-28.

69. De Palma FDE, Del Monaco V, Pol JG, Kremer M, D'Argenio V, Stoll G, et al. The abundance of the long intergenic non-coding RNA 01087 differentiates between luminal and triple-negative breast cancers and predicts patient outcome. Pharmacol Res. 2020;161:105249.

\section{Publisher's Note}

Springer Nature remains neutral with regard to jurisdictional claims in published maps and institutional affiliations.

Ready to submit your research? Choose BMC and benefit from:

- fast, convenient online submission

- thorough peer review by experienced researchers in your field

- rapid publication on acceptance

- support for research data, including large and complex data types

- gold Open Access which fosters wider collaboration and increased citations

- maximum visibility for your research: over 100M website views per year

At BMC, research is always in progress.

Learn more biomedcentral.com/submissions 